\title{
Les cosaques aux Champs-Elysées. L'occupation de la France après la chute de Napoléon
}

\section{Marie-Cécile Thoral}

\section{(e) OpenEdition \\ 1 Journals}

Édition électronique

URL : https://journals.openedition.org/ahrf/7903

DOI : $10.4000 /$ ahrf.7903

ISSN : 1952-403X

Éditeur :

Armand Colin, Société des études robespierristes

Édition imprimée

Date de publication : 1 décembre 2006

Pagination : 171-174

ISSN : 0003-4436

Référence électronique

Marie-Cécile Thoral, « Les cosaques aux Champs-Elysées. L'occupation de la France après la chute de Napoléon », Annales historiques de la Révolution française [En ligne], 346 | Octobre/Décembre 2006, mis en ligne le 10 juillet 2008, consulté le 22 avril 2022. URL : http://journals.openedition.org/ahrf/7903 ; DOI : https://doi.org/10.4000/ahrf.7903

Ce document a été généré automatiquement le 22 avril 2022.

Tous droits réservés 


\title{
Les cosaques aux Champs-Elysées. L'occupation de la France après la chute de Napoléon
}

\author{
Marie-Cécile Thoral
}

\section{RÉFÉRENCE}

Jacques Hantraye, Les cosaques aux Champs-Elysées. L'occupation de la France après la chute de Napoléon, Paris, Belin, 2005, 303 p., ISBN 2-7011-4009-9, $25 €$.

1 Dans ce livre, tiré d'une thèse de doctorat soutenue en 2001 sous la direction d'Alain Corbin, Jacques Hantraye retrace l'histoire des deux phases d'occupation militaire de la France par les Alliés en 1814 et 1815 à la suite de la chute de Napoléon, en choisissant pour cela un département " moyen ", intermédiaire entre les départements frontaliers les plus exposés et les départements du centre les plus épargnés : la Seine-et-Oise. Il offre ainsi une contribution importante à l'histoire de la transition post-napoléonienne, et à l'histoire contemporaine de l'Europe en général. Le sujet est riche, original, intéressant et surtout très peu étudié par les historiens jusqu'à présent.

2 Le sujet d'ensemble du livre, tel qu'il est défini par l'auteur dans son introduction (faire l'étude d'un département en guerre) est un peu trop descriptif. L'absence d'une réelle problématique d'ensemble, qui fournirait la trame du livre et la matière à une démonstration, rend en effet la lecture du livre et la compréhension de la nature et de la portée historiques des événements difficiles. Il n'en reste pas moins que l'étude est sérieuse, bien appuyée sur des sources d'archives et des sources imprimées variées (journaux locaux, mémoires de militaires Alliés), et que l'auteur apporte des renseignements tout à fait intéressants sur le déroulement de ces deux périodes de guerre et d'occupation.

Le plan thématique choisi, assez pertinent, correspond aux trois grandes questions posées par l'auteur dans son introduction, trois problématiques centrales à l'étude des 
relations entre guerre et société mais isolées, non reliées l'une à l'autre : les « violences de guerre ", la vie quotidienne des civils pendant cette période, et les marques de la "présence des occupants » dans la société française.

4 L'auteur commence son étude par une analyse des causes, des motivations et des formes de la "violence de guerre" au sens large (actes de violence commis sur des civils pendant la guerre et l'occupation). Un ensemble de causes ou de raisons potentielles de la violence des Alliés est successivement passé en revue : le sentiment national et la volonté de revanche (peut-être écartés un peu trop vite), la peur ressentie par les Alliés dans un pays étranger et surtout face à une population vue comme menaçante, la consommation de drogue et d'alcool, la fatigue, la crainte d'une instabilité politique et d'une impuissance des autorités françaises, la volonté de marquer le territoire et de montrer aux civils français qui détient désormais le pouvoir (la violence apparaissant alors comme un élément d'une domination symbolique)... L'auteur conclut ce chapitre par l'affirmation d'une sorte de «seuil » de la violence qui ne serait pas, ou peu, franchi par les Alliés, d'une violence limitée, ce qui remet en cause l'idée généralement reçue d'une violence exacerbée des Alliés (notamment des Prussiens et des Russes). Il livre ainsi, dans ce premier chapitre, une lecture originale, faisant la part belle aux représentations et à la psychologie collective, d'un élément central dans l'étude de l'histoire culturelle de la guerre, de l'histoire des civils dans la guerre : les violences hors du champ de bataille.

5 Le deuxième chapitre de cette première partie est consacré à l'étude du comportement des Français face à la menace de guerre et à l'invasion. Napoléon et ses collaborateurs ont une attitude hésitante et pleine de contradictions face à l'appel aux civils pour une aide militaire. Ils commencent par demander une aide limitée (surveiller les mouvements ennemis notamment) et encadrée par les notables, sans pour autant autoriser l'armement du peuple, par crainte d'une dérive. Face à l'avancée des Alliés, les gouvernants se résolvent finalement à demander l'aide militaire de tous les civils, peuple et notables, et décident la levée en masse le 5 mars 1814. Trop tard selon Jacques Hantraye, qui voit dans ces atermoiements politiques l'une des causes de la défaite française en 1814. Instruits par l'expérience, les gouvernants des Cent-Jours en appellent à une mobilisation générale dès le 3 mai 1815. Jacques Hantraye affirme cependant que les civils ont été assez réticents face à cet appel, malgré les efforts des maires pour les engager à partir. Pourtant, plus de $90 \%$ du quota de gardes nationaux mobilisés pour le département de Seine-et-Oise en juin 1815 part au combat. La mobilisation n'est donc peut-être pas si inefficace. Jacques Hantraye évoque ensuite, à juste titre, le rôle essentiel des gardes nationaux sédentaires dans la protection des communautés pendant la guerre et pendant l'occupation. Cette importance de la défense communautaire se retrouve aussi dans la réaction des civils face aux Alliés sous l'occupation. Les habitants de Seine-et-Oise ne se révoltent pas, dans l'ensemble, face aux militaires alliés, à la fois par impuissance (après le choc de l'invasion qui les aurait tétanisés) et par prudence (face à une présence ennemie massive et diffuse). Les quelques cas de résistance collective sont surtout des actes de protection des biens de la communauté (notamment les productions fourragères) face aux réquisitions des occupants. Jacques Hantraye s'attache ensuite à essayer de montrer le poids de la présence étrangère. Si les effets de la guerre et de l'occupation sur les propriétés sont peu importants (peu de maisons détruites par suite des combats, peu d'incendies, quelques ponts détruits), les réquisitions sont durement ressenties par les civils, d'autant plus que l'abondance des doses alimentaires données aux soldats étrangers et 
le gaspillage qui accompagne parfois ces réquisitions heurtent les pratiques frugales de la paysannerie. Autant les pillages ne concernent pas toutes les communes du département (environ $20 \%$ des communes sont épargnées), autant les réquisitions sont supportées par toutes les communes, ou presque (environ $2 \%$ des communes épargnées). Le poids de la présence étrangère, pour la plupart des civils, c'est donc le prélèvement quotidien d'une part de la production agricole pour la nourriture des militaires étrangers.

6 L'auteur passe ensuite, dans la deuxième partie, à une étude de la société française sous l'occupation. Dans le premier chapitre, il évoque la réaction des habitants face à l'invasion puis à l'occupation. Dans un premier temps, face au danger de l'invasion qui se rapproche, la peur et des comportements de protection semblent l'emporter. Les habitants cachent une partie de leurs biens pour éviter qu'ils ne soient pillés, et un certain nombre d'entre eux, plusieurs milliers ou dizaines de milliers selon Jacques Hantraye, fuient pour tenter de se mettre à l'abri. Cependant, ces migrations temporaires n'ont pas une très grande ampleur, d'une part, parce que les habitants ne s'éloignent pas beaucoup de leur commune, d'autre part, parce que les retours se font rapidement après l'occupation. Si la guerre bouleverse le quotidien, les civils s'adaptent progressivement. Peu de temps après les débuts de l'occupation, les habitants reprennent leurs activités professionnelles (récoltes pendant l'été 1815) ou de loisir (réouverture de certaines maisons de campagne), leur routine quotidienne.

7 La guerre et l'occupation ont aussi des effets sur l'administration du département. Jacques Hantraye apporte des éléments intéressants sur le fonctionnement administratif sous l'occupation, notamment dans le domaine des pratiques administratives (augmentation du travail, importance des questions militaires dans le travail quotidien des administrateurs civils, établissement de formulaires spécifiques à cette période...). Il passe cependant un peu rapidement sur les effets de ces événements sur le personnel administratif (la majorité des fonctionnaires et administrateurs restent-ils en poste ou ont-ils fui ?), sur les épurations (évoquées pour quelques cas ponctuels : préfets, quelques maires...) et sur les relations entre les officiers prussiens établis à Versailles et les autorités françaises (à peine une demie-page, p. 153, pour évoquer la soumission des autorités françaises aux ordres des Prussiens).

8 La troisième partie du livre est consacrée à une étude des formes de la " présence des occupants" dans la société. Après avoir évoqué les traces matérielles éphémères (spectacle des transports de troupe, «signaux " prussiens pour faciliter les transports et le repérage, établissement de bâtiments militaires pour les occupants : infirmeries, corps de garde ; circulation des militaires pour visiter tel ou tel monument : « tourisme militaire »...) ou plus durables (présence, plusieurs mois après la fin des combats, d'objets rappelant le passage des Alliés : boulets de canon, armes, pièces de monnaie...), l'auteur aborde la question des relations entre occupants et civils français grâce à trois éléments d'étude : l'attitude des militaires occupants par rapport à la société française (l'installation définitive de certains d'entre eux en France après la fin de l'occupation montre que certains se sont bien intégrés), les représentations collectives françaises des Alliés (avec un exemple : l'élaboration de la figure du Cosaque) et enfin l'attitude des Français par rapport aux militaires occupants blessés ou morts, attitude qui est, selon Jacques Hantraye, révélatrice de l'attitude générale des civils français par rapport aux occupants (l'inscription des morts Alliés dans les registres des morts français, et le partage symbolique de la terre sacrée du cimetière avec les occupants montrant, selon 
l'auteur, l'acceptation de la présence étrangère et une certaine estime pour les militaires morts au combat). Le passage le plus intéressant, dans cette troisième partie, est l'étude du regard croisé entre peuples de nationalités différentes dans le chapitre sur les Cosaques. L'auteur, en digne élève d'Alain Corbin, montre avec beaucoup de finesse et de sensibilité l'évolution des stéréotypes nationaux au cours de cette période de contacts multipliés entre civils français et militaires étrangers. Certains Français, en observant la conduite des Russes, se déprennent de leurs préjugés, d'autant plus qu'un déclassement dans la hiérarchie des nationalités violentes et brutales se fait souvent à cette période (les Prussiens étant vus comme plus violents que les Russes). L'évocation de militaires russes cherchant à savoir ce que les étrangers, Allemands ou Français, pensent d'eux (en regardant les gravures, les livres, les pièces de théâtre) est très intéressante et donne à réfléchir sur l'influence de ces deux périodes de guerre et d'occupation sur l'affirmation des sentiments nationaux en Europe au XIX ${ }^{\mathrm{e}}$ siècle, sujet non abordé dans ce livre, mais qui en serait un prolongement intéressant.

Ce livre est une contribution à l'histoire d'un événement méconnu mais essentiel, et un bel exemple de l'intérêt d'une approche culturelle de faits politiques et militaires. L'auteur évoque avec sensibilité les sentiments et les émotions des acteurs du conflit et donne au lecteur une vision colorée et fidèle de l'ambiance générale de la période. L'intérêt de l'étude est un peu compromis par l'absence d'une réelle problématique d'ensemble, mais le livre contient des données intéressantes et des idées originales. 